\title{
Mobile-based Decision Support System for Poultry Farmers: A Case of Tanzania
}

\author{
Martha Shapa ${ }^{1}$, Lena Trojer ${ }^{2}$, Dina Machuve ${ }^{3}$ \\ School of Computational and Communication Science and Engineering \\ Nelson Mandela African Institution of Science and Technology, Arusha, Tanzania ${ }^{1,3}$ \\ Department of Technology and Aesthetics Blekinge Institute of Technology, Karlshamn, Sweden ${ }^{2}$
}

\begin{abstract}
Poultry farms in Tanzania are characterized by inadequate management practices which are mainly caused by the lack of adequate systems to guide the small-scale poultry farmers in decision making. It is well-established that information is a key factor in making effective decisions in numerous sectors including poultry farming. Furthermore, various researchers have identified the use of mobile decision support tools to be an effective way of aiding farmers in making informed decisions. In this paper, we present a mobile-based decision support system that will aid rural and small-scale poultry farmers in Tanzania to obtain reliable information that is crucial for making proper decisions in their farming activities. In this context, a mobile-based decision support system was achieved through a mobile application integrated with a chatbot assistant to provide a solution to various poultry farming-related problems and simplify their decision-making process. We used a data-driven approach towards developing an informational chatbot assistant for Android smartphones that is capable of interacting with small-scale poultry farmers through natural conversations by utilizing the RASA framework.
\end{abstract}

Keywords-Decision support system; chatbot; mobile application; poultry farming; data-driven approach

\section{INTRODUCTION}

Poultry farming is one of the prominent agricultural sectors dealing with the keeping of various domestic birds to produce eggs and meat for consumption and trade [1]. Similarly, it is one of the important agricultural areas for generating income for farmers in Tanzania. Studies indicate that there are approximately 36.2 million chickens in Tanzania, of which approximately $95 \%$ are local chickens reared by rural households [2]. Over the past decade, the growth of poultry production has accelerated, due to the rapid urbanization and increase in demand for poultry products, which include chicken meat and eggs [3]. Poultry farming has a direct impact on the farmers and has gained a notable attraction among entrepreneurs and women. Generally, it is the source of the poultry farmer's family income and protein. In this sector, women constitute the majority of poultry farmers as they constitute over $80 \%$ of the farming population in Tanzania [4].

The majority of small-scale farmers in Tanzania rely on poultry farming as their major source of income [5]. However, poultry production is hindered by several challenges including unreliable markets, poultry diseases, scarce inputs, and shortage of timely extension information due to scarcity of extension officers as well as distant locations for consultation [1]. It has been observed that rural and small-scale poultry farmers rely mainly on unreliable sources of information for poultry management such as word of mouth from family members, neighbors, and friends with previous poultry keeping experience due to the lack of adequate systems to guide them in decision making [6]. Moreover, information is very important in the development of poultry farming and agriculture at large, therefore the information obtained from unreliable sources may lead to underdevelopment of this sector, especially to rural, peri-urban, and small-scale poultry farmers [1].

Technology advancement plays a great role in the agricultural sector development, including poultry farming. It has been argued that the use of mobile decision support tools is an effective way of aiding farmers to make informed decisions [6]. Due to an increase in the use of mobile communication technologies in different sectors in the country [7], this study will contribute and improve proper information attainment for poultry farming, and aiding farmers to make informed decisions.

Along with the technological advancement and the increase in the information-seeking behavior of the small-scale poultry farmers in Tanzania [6], this study will play a great role in aiding the small-scale poultry farmers attain crucial poultry farming information in time and make informed decisions. A mobile-based decision support system is achieved through a mobile application with a chatbot assistant that provides a solution to various poultry farming-related problems and simplifies their decision-making process. Furthermore, the conversational assistant, chatbot, is a modern human-computer interaction technology that was introduced in the 1960s when the earliest chatbot ever was developed [8], [9], and gained popularity in 2016. It has been argued that chatbot is one of the most advanced and effective ways to provide information and facilitate the decision-making process in various sectors [10]. According to [8], [11], chatbots can be used to aid farmers by providing information and solutions through responding to poultry farming-related problems and facilitate their decisionmaking process in poultry farming.

This paper introduces a decision support mobile application, for providing poultry farming-related information to small-scale poultry farmers in Tanzania. For this purpose, the mobile application was developed using Android Studio, and integrated with a chatbot.

The remainder of this paper is organized as follows: Section II presents the related works carried out in solving 
various farm-related problems. Section III demonstrates the methodology used in the requirements gathering, the approach and tools used in the development of the mobile-based Decision Support system. Section IV discusses the results of the proposed system, and followed by conclusion in Section V.

\section{RELATED WORKS}

Various researchers have revealed that the use of mobilebased conversational assistants is an effective way to aid farmers with farm-related information and problem-solving. In the early days after the first chatbot was developed, chatbots had limited effectiveness and maintained a simple conversational flow. As the research progresses, recent chatbots are capable of understanding the context of the user and the flow of the conversations and provide a suitable response. Several studies have been conducted to develop chatbots that will assist farmers in solving their farm-related problems.

Jain et al. [11] designed a chatbot called FarmChat that aims to meet the information needs of farmers in rural India. The system offers information to the farmers by answering their farming-related queries. It was developed using the IBM Watson Assistant and consists of two interface modalities: Audio-only, and Audio+Text [11]. The study was conducted with 34 potato farmers in rural India and indicated that the chatbot offered satisfying information that supported them [11]. Thus, the authors suggested that conversational assistance delivered through smartphones could be an effective way to improve the information accessible to people with limited literacy in rural areas.

Arora et al. [8] developed an interactive chatbot named Agribot, that assists farmers in problem-solving, crop disease detection, and weather prediction. They developed the chatbot using sequence-to-sequence learning, an approach that allows the model to learn the mapping between questions and their suitable response [8]. The authors suggested that the chatbot could be more generalized in terms of conversations if the model is trained in a massive amount of data-points [8].
Fue et al [12] developed an agro-advisory web and mobilebased system called 'Ushaurikilimo' that allows farmers to request advisory services from an agriculture extension officer using either the web or mobile phone [12]. 'Ushaurikilimo' operates in Tanzania. It is a two-way communication platform between farmers and experts [12]. It functions by allowing farmers to ask questions through SMS and get a response from the agricultural expert [12]. The platform allows farmers to ask for advice on agricultural-related issues like; farm management, livestock keeping, marketing information, and aquaculture [12], whereby it depends on the presence of the experts in order for the farmers' problems to be solved. The proposed system aims at solving this gap.

\section{METHODOLOGY}

The methodological approach we used in the development of a mobile-based Decision Support system for small-scale poultry farmers in Tanzania in this study is the utilization of Android Studio in the mobile application development and a Rasa framework in the development of a chatbot that will aid small scale poultry farmers in Tanzania by giving answers to their poultry-related problems and help them make an informed decision in their poultry management practices.

As illustrated in Fig. 1, when the small-scale poultry farmer types his or her poultry-related query in the mobile application's chat window and sends it, the text is fed into Rasa NLU through the Application Programming Interface (API). An API software intermediary allows the farmer's mobile application and our assistant to communicate by delivering the requests from the poultry farmer to the chatbot assistant and delivering the response back to the poultry farmer [13]. After the Rasa NLU receives the text message from the poultry farmer in form of a natural human language, performs intent classification, entity extraction, and converts it into the form of structured data that our chatbot assistant could understand what the farmer is saying.

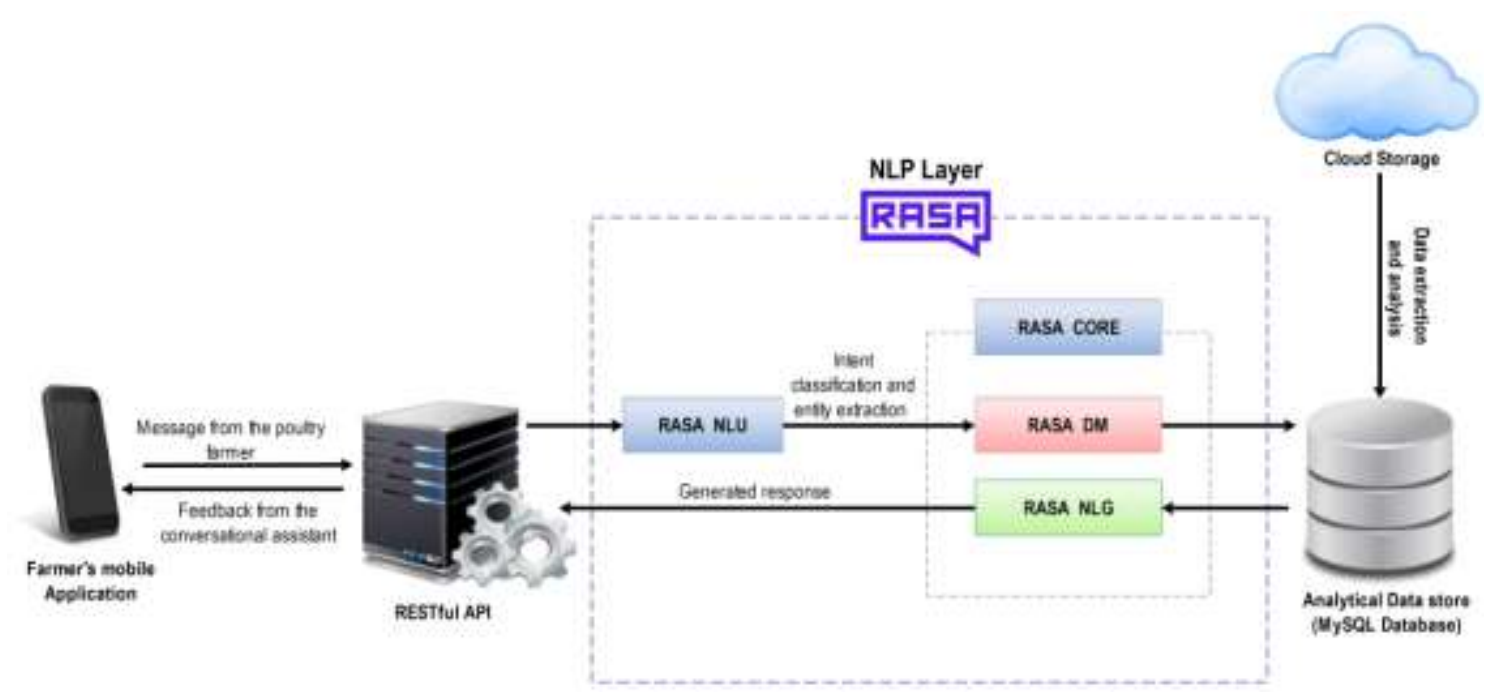

Fig. 1. A Proposed Conceptual Framework 
Furthermore, based on the intents and entities, the Rasa Core takes structured inputs from the Rasa NLU through the dialogue management and predicts the next desirable action using a probabilistic model called Long short-term memory (LSTM) neural network [14], through the LSTM based supervised learning (SL) and Reinforced learning (RL) rather than the if/else statement [14]. Reinforced Learning (RL) is used to improve the next best action. Therefore, Rasa Core performs Dialogue Management by keeping track of the conversation and decide the next set of actions to be performed by the chatbot [15]. An action can be a simple utterance that means sending a feedback message to the poultry farmer, or an arbitrary function to execute. After the action has been executed, it passes a tracker instance to use any relative information collected in the dialogue history and previous actions [15].

Additionally, Rasa Core has high Natural Language Generation (NLG) capabilities [16], [17]. Therefore, upon retrieval, the Rasa Core uses its NLG capabilities to prepare a natural language human-like response to the poultry farmer based on the intent and context information returned from the Rasa NLU [17]. The dialogue manager generates raw responses that will be passed to the Natural Language Generator component that refines the text response and construct the understandable text responses in natural human language in machine representation. NLG process converts structured data into text, therefore it generates an appropriate response that a human can understand [16]. Finally, the generated feedback is sent back to the farmer's mobile application User interface via the API.

\section{A. Data Collection and Requirements Gathering}

An exhaustive literature review was conducted to identify and assess the information management requirements of smallscale poultry farmers. The identified information management requirements as summarized in Table I. Includes literature, frequency, and percentage of frequently asked questions by poultry farmers in attempts to attain proper information for their proper poultry management practices. The identified information management requirements of poultry farmers according to Table I, includes:

1) Chicken health: From an exhaustive literature review, various studies have identified that the majority of the smallscale poultry farmers in Tanzania are facing challenges in maintaining the good health of their poultry [6]. The studies show that small-scale poultry farmers in Tanzania face many health-related problems in their poultry farms and hence tend to seek various information concerning poultry health from various sources of information, mostly unreliable sources. The health information they mostly seek includes; disease control, diagnosis of the chicken diseases, the transmission of diseases, and vaccination of chickens against different diseases [1], [6].

2) Chicken feeds: One of the most important information needs that small-scale poultry farmers in Tanzania seek includes chicken feeds information [1]. The small-scale poultry farmers frequently ask questions about the availability of the chicken feeds, Types of feeds for their chicken types, amount of feeds per chicken, and feed formulation and preparation for the chicken feed, to maintain the proper production [6].

3) Chicken breeds: According to [6], poultry farmers are interested in knowing the different types of chicken breeds for various purposes before starting up a poultry farm. The factors that the majority of small-scale poultry farmers consider include; diseases resistant breed types, breed types that are best for commercial purposes, and breed types for egg production [6]. The poultry farmers also seek general information on chicken breeds to help them in proper keeping of a particular chicken breed, and rearing techniques of different types of chicken breeds.

4) Egg production: Among the frequently asked questions by many small-scale poultry farmers in Tanzania includes questions about egg production, particularly for commercial purposes. [6], [18] highlights that small-scale poultry farmers seek information about egg production, which includes improving the quality and production of eggs, proper storage of eggs, and need to know the best method for incubation of eggs [6].

5) Housing: Various studies have highlighted that majority of the startup small-scale poultry farmers tend to seek information on the housing of the chicken shelter before they start practicing poultry farming [6]. The farmers are more interested in attaining the information about the startup capital for poultry farming, characteristics of the chicken house and how to build it regarding the geographical position of the farm, and size of the chicken house for a certain number of chickens [1].

The identified information management requirements of small-scale poultry farmers through literature review were the requirements gathered and used in the development of the mobile application for poultry farming data collection, and training of our chatbot assistant to offer reliable poultry farming information that will enable the small-scale poultry farmers to make informed decisions. The information management requirements gathered helped in the identification and attainment of the 200 sample questions and answers for poultry farming-related problems, these sample questions and answers were used in training the NLU model of our chatbot assistant.

\section{B. Approach for Mobile Application Development}

This study aims at the development of a mobile-based decision support system, which involves the development of a mobile application. The approach used in the development of the mobile application is the Android studio with JAVA programming language [20]. This approach was chosen due to the reasons that the Android mobile operating systems are widely used by the targeted users [21]. Android Studio was selected because it is suitable for the development of Androidbased applications for smartphones with Android operating systems [20]. The minimal version of the Android operating system supported by the developed mobile application is Jelly Bean 4.3, this was selected because it is more inviting for the average user. 
TABLE I. INFORMATION MANAGEMENT REQUIREMENTS FOR POULTRY FARMERS

\begin{tabular}{|c|c|c|c|c|c|}
\hline Number & Literature Author & Sample Space & Information need & Frequency & Percentage \\
\hline \multirow{4}{*}{1.} & \multirow{4}{*}{ Grace Msoffe et al. [6] } & \multirow{4}{*}{187} & Disease control & 187 & 100 \\
\hline & & & Breeds and breeding & 78 & 41.7 \\
\hline & & & Housing and shelter & 56 & 29.9 \\
\hline & & & Feeding and nutrition & 47 & 25.1 \\
\hline \multirow{3}{*}{2.} & \multirow{3}{*}{ Temba B et al. [1] } & \multirow{3}{*}{160} & Poultry diseases & 112 & 70 \\
\hline & & & Poultry nutrition & 92 & 57.5 \\
\hline & & & Housing & 92 & 57.5 \\
\hline \multirow{4}{*}{3.} & \multirow{4}{*}{ Benjamin Folitse et al. [18] } & \multirow{4}{*}{150} & Disease management & 145 & 96.7 \\
\hline & & & Eggs production & 114 & 76 \\
\hline & & & Feeding and nutrition & 112 & 74.7 \\
\hline & & & Shelter & 108 & 72 \\
\hline \multirow{3}{*}{4.} & \multirow{3}{*}{ Jotshana Khobragade et al. [19] } & \multirow{3}{*}{60} & Health and disease control & 56 & 93.34 \\
\hline & & & Feeding management & 55 & 91.67 \\
\hline & & & Housing management & 53 & 88.34 \\
\hline
\end{tabular}

\section{Natural Language Processing Layer}

The methodological approach we used in this study is the utilization of a Rasa framework in the development of a chatbot that will aid small scale poultry farmers in Tanzania by giving answers to their poultry-related problems and help them make an informed decision in their poultry management practices. The Rasa framework is an open-source machine learning framework for building contextual conversational assistants called chatbots, these assistants consist of two components which are Rasa NLU and Rasa Core [22].

1) Rasa NLU: This is the Rasa's desirable library for Natural Language Understanding that performs intent classification and entity extraction [17]. It takes user inputs in a simple unstructured human language and extracts structured semantic information in the form of intents and entities [17], [23]. Intents are labels that are attached to each user's input based on the overall goal of the user's message, and entities are pieces of information that our conversational assistant may need in a certain context. Furthermore, Rasa NLU is treated as the ear of the chatbot, because it teaches the chatbot to understands the inputs of the user [22].

2) Rasa Core: This is a framework for machine learningbased contextual decision making so-called the brain of our assistant because it predicts how our assistant will respond based on a specific state of the conversation as well as the context [17], [22]. It learns by observing the pattern from example conversational data between the user and the assistant also called stories.

Rasa Core is responsible for Dialogue management (Rasa DM). In Rasa, Dialogue management learns the patterns of the conversations from the example conversational data using Machine learning and predict how our assistant should respond in a specific situation based on the history of the conversation and the context [15], [17]. In Dialogue management the training Data for our conversational assistant is called stories, these are example conversations between the small-scale poultry farmer and our assistant, written in a specific format. This format includes expressing the user inputs as relative intents and entities, the same way as they were expressed in the NLU training Data, while the responses of our assistant were expressed as action names [15].

Furthermore, Rasa Core has high NLG capabilities that enable the chatbot to intelligently know the exact and clear response that is to be generated for a corresponding user message [17].

\section{Training of the Rasa Conversational System Model}

In the training of our conversational model, both Rasa NLU and Rasa Core use human-readable training data formats. Rasa NLU requires a list of utterances that are annotated with intents and entities for training our chatbot assistant [15]. We used both JSON structure and markdown format in the training of our chatbot assistant [15]. Using the Rasa NLU pipeline, we prepared a training data set to classify the intents and extract the entities. The training data includes several intents: greet, goodbye, chicken_feeds, chicken_breeds, eggs_production, chicken_diseases, and chicken_shelter. We use about 200 sample questions and answers with marked entities to train the Rasa NLU [15], [16].

In addition to the supervised learning, Rasa Core supports a machine teaching approach whereby, the actions made by the system can be corrected by the developers, we used this approach in generating training data and inspecting the space of credible conversations efficiently [15], [24]. The Training data used in training the Rasa Core are known as stories, these are the sample conversations between the user and our chatbot assistance [24]. Furthermore, Rasa core's Machine Learning libraries give it the capability of learning from the previous conversations between the user particularly the poultry farmer, and our chatbot assistant [17]. 


\section{1) Markdown training data format example}

\#\# intent:chicken_diseases

- How do you treat a chicken's skin wounds?"

- Can I keep sick poultry together with normal ones?

- What is causing your hen's swollen foot and her limping?

\#\# intent:egg_production

- How often do [layers](flock_name) lay eggs?

- When will my hens start laying?

- How long do chickens lay eggs?

- Why do some eggs have soft shells or no shells?

\#\# intent:greet

- hi

- hey

- hello

2) JSON training data format example

"rasa_nlu_data": \{

"common_examples": [

\{

"text": "Hello",

"intent": "greeting",

"entities": []

\} ,

\{

"text": "How do you treat a chicken's skin wounds?",

"intent": "chicken_diseases",

"entities": []

\}

\{

ones?",

"text": " Can I keep sick poultry together with normal

"intent": " chicken_diseases",

"entities": []

\}

\{

"text": " When will my hens start laying?",

"intent": "egg_production"

\}

],

"regex_features": [],

"entity_synonyms": []

\}

\}

\section{RESULTS AND DISCUSSION}

Based on the study described, a mobile-based Decision Support system for poultry farmers was implemented. The developed Android-based mobile application for poultryrelated data storage and poultry farming-related information provision was integrated with our chatbot assistant, for the aim of aiding small-scale poultry farmers with reliable information for productive management practices.

The developed mobile application as illustrated in Fig. 2, consists of five modules; Consultation module, Information portal, events, new records, and my records. The information portal contains various general information that the small-scale poultry farmer will require in poultry farming.

The new records module allows the small-scale poultry farmer to keep records of his or her day to day flock management activities, feed management, which includes the amount and type of feeds the farmer offers to the chicken. Medication records, the farmer will be able to keep records of all medications provided to the chickens, which includes the vaccination record, vitamins provision, and general medications offered to the farmer's flock, lastly are the records of the finances, the farmer can keep records of the sales and expenditures of the poultry farm. The farmer can view the farming records and keep track of the development of the poultry farm on a timely basis.

The recorded poultry farming data are stored in the cloud storage, together with the stored previous conversations are used by our chatbot assistant in responding to various poultryrelated questions, and advice the farmer regarding the particular poultry farmer's farm and flock condition. This Data-driven Approach used in the development of our mobilebased decision support system makes our system intelligent enough to help the poultry farmers in their decision making for productive poultry management practices [25].

The developed mobile application consists of a consultation module. The consultation module is the chatbot assistant that responds to the poultry farmers' questions concerning chicken health, chicken feeds, chicken eggs production, and the chicken breeds. The chatbot assistant was trained to offer consultation to the small-scale poultry farmers regarding the most common poultry-related problems that mostly face them, and help the poultry farmer make proper decisions in practicing poultry farming. The consultation chat between the user and our chatbot assistant is illustrated in Fig. 3. 


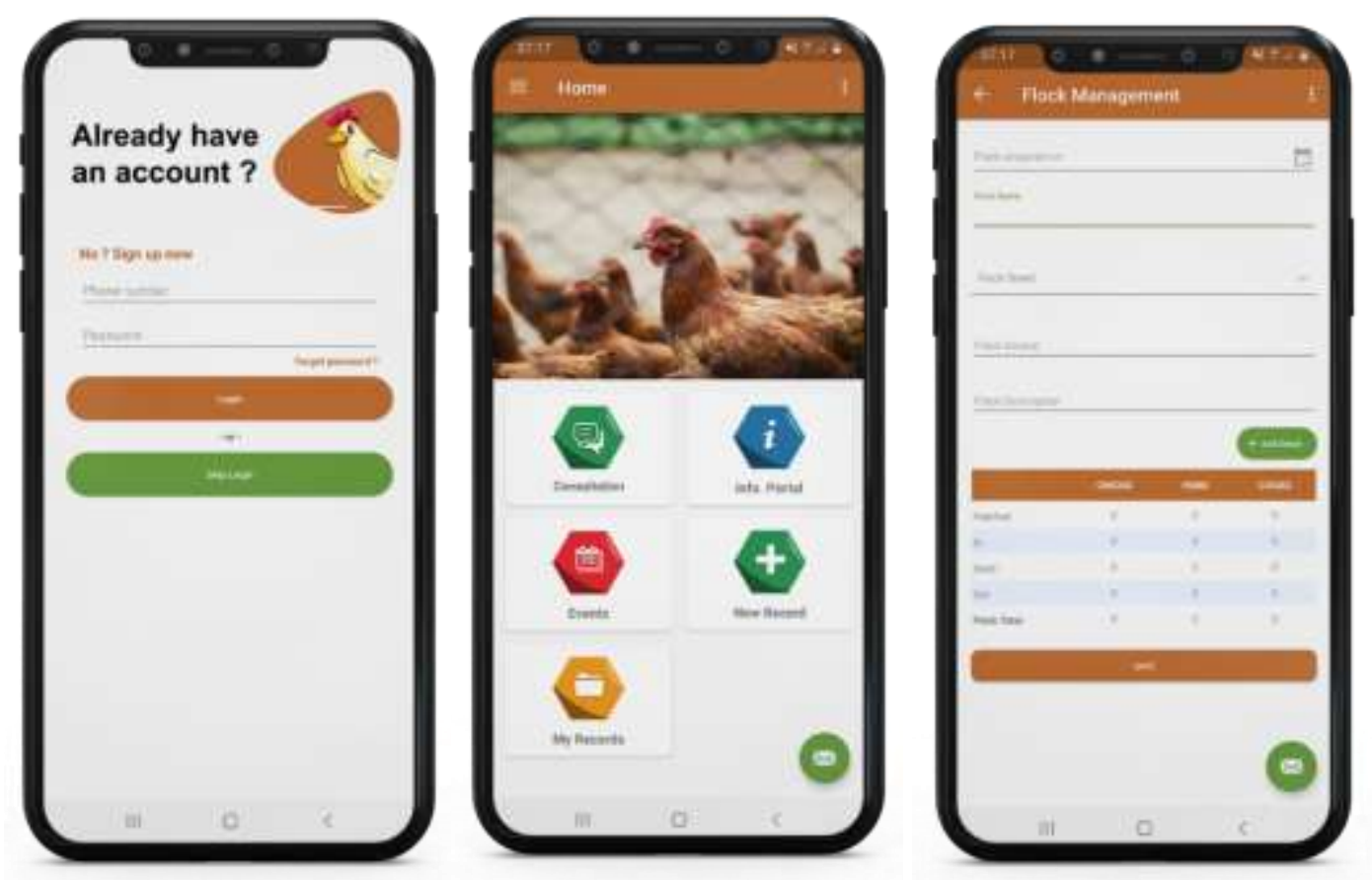

Fig. 2. Poultry Farmer's Mobile Application.
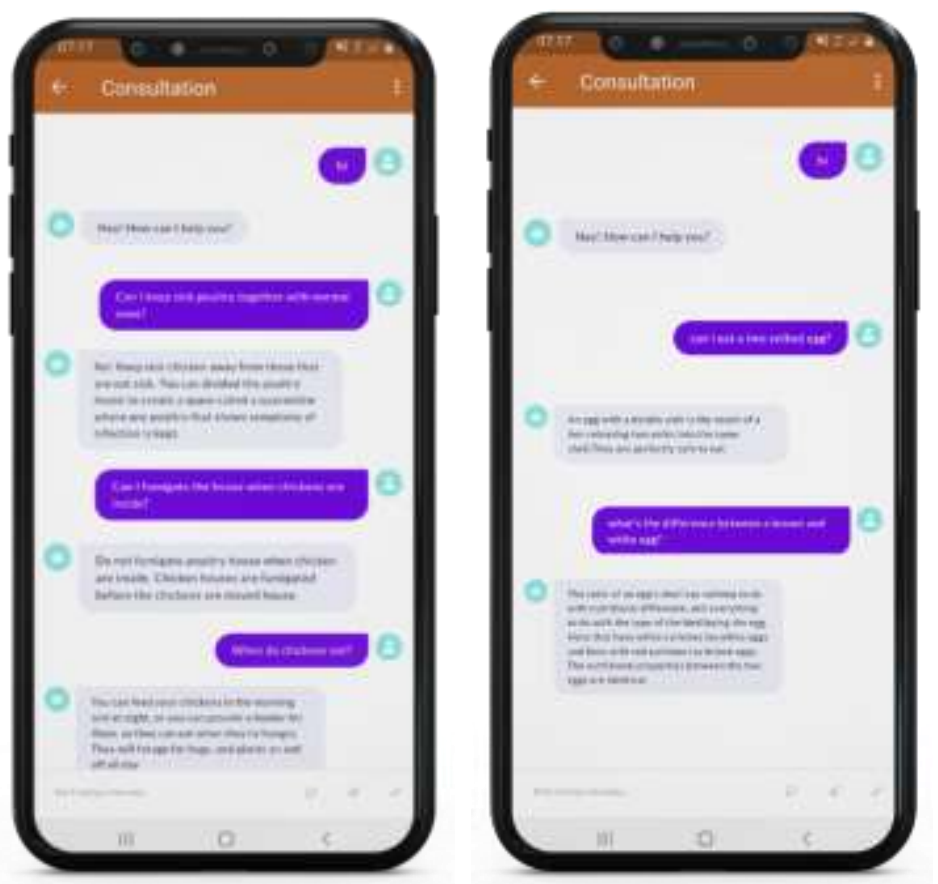

Fig. 3. Conversations on the Consultation Module.

\section{CONCLUSION}

This paper has presented an exploratory study using a mobile conversational agent-based interaction to facilitate intelligent decision support to the small-scale poultry farmers during a consultation with our assistant. We implemented the proposed mobile-based decision support system for poultry farmers via an interactive chatbot assistant using a Rasa framework, and an Android-based mobile application using Android studio.
Future work focuses on the validation of the mobile application developed to ensure the extent to which this study contributes in aiding farmers with the crucial poultry farming information that they frequently seek for making informed decisions. This process will involve user acceptance testing, to testing, by letting the small-scale poultry farmers use the mobile application by storing their poultry-related data and consult our chatbot assistant via the mobile application. 


\section{ACKNOWLEDGMENT}

The authors would like to acknowledge the support in this study from the Organization for Women in Science for the Developing World (OWSD) Early Career Fellowship Programme (Agreement number 506575) and the African Development Bank (AfDB).

\section{REFERENCES}

[1] B. A. Temba, F. K. Kajuna, G. S. Pango, and R. Benard, "Accessibility and use of information and communication tools among farmers for improving chicken production in Morogoro municipality, Tanzania," Livest. Res. Rural Dev., vol. 28, no. 1, 2016.

[2] A. Saleque, A. Jabeen, and S. M. Real, "Small Scale Poultry Rearing in Tanzania - Subsistence to Surplus Production for Increase Income and Improve Food and Nutrition Security.," pp. 1-7, 2016.

[3] A. Vernooij, M. N. Masaki, and D. Meijer-Willems, "Regionalisation in poultry development in Eastern Africa," 2018, [Online]. Available: www.wageningenUR.nl/livestockresearch.

[4] E. L. Isaya, R. Agunga, and C. A. Sanga, "Sources of agricultural information for women farmers in Tanzania," Inf. Dev., vol. 34, no. 1, pp. 77-89, 2018, doi: 10.1177/0266666916675016.

[5] A. Ali et al., "Backyard poultry farming empowering women for doubling farmers ' income," vol. 8, no. 2, pp. 1138-1143, 2020.

[6] G. Msoffe, A. Chengula, M. J. Kipanyula, M. R. S. Mlozi, and C. A. Sanga, "Poultry Farmers' Information needs and Extension advices in Kilosa, Tanzania: Evidence from Mobile-based Extension, Advisory and Learning System (MEALS)," Libr. Philos. Pract., vol. 2018, no. February, 2018.

[7] BMI, "Tanzania Telecommunications Report," p. 62, 2016.

[8] B. Arora, D. S. Chaudhary, M. Satsangi, M. Yadav, L. Singh, and P. S. Sudhish, "Agribot: A Natural Language Generative Neural Networks Engine for Agricultural Applications," 2020 Int. Conf. Contemp. Comput. Appl. IC3A 2020, pp. 28-33, 2020, doi: 10.1109/IC3A48958.2020.233263.

[9] M. H. Wen, “A conversational user interface for supporting individual and group decision-making in stock investment activities," Proc. 4th IEEE Int. Conf. Appl. Syst. Innov. 2018, ICASI 2018, pp. 216-219, 2018, doi: 10.1109/ICASI.2018.8394571.

[10] O. Chukhno and N. Chukhno, "A chatbot as an environment for carrying out the group decision making process," vol. i, pp. 15-25, 2019.

[11] M. Jain, P. Kumar, I. Bhansali, Q. V. Liao, K. Truong, and S. Patel, "FarmChat: A Conversational Agent to Answer Farmer Queries," Proc. ACM Interactive, Mobile, Wearable Ubiquitous Technol., vol. 2, no. 4, pp. 1-22, 2018, doi: 10.1145/3287048.

[12] K. Fue, A. Geoffrey, M. Mlozi, S. Tumbo, R. Haug, and C. Sanga, "Analyzing usage of crowdsourcing platform Ushaurikilimo' by pastoral and agro-pastoral communities in Tanzania," 2016.
[13] R. Gunawan, I. Taufik, E. Mulyana, O. T. Kurahman, M. A. Ramdhani, and Mahmud, "Chatbot Application on Internet of Things (IoT) to Support Smart Urban Agriculture," Proceeding 2019 5th Int. Conf. Wirel. Telemat. ICWT 2019, pp. 3-8, 2019, doi: 10.1109/ICWT47785.2019. 8978223.

[14] Y. Windiatmoko, A. F. Hidayatullah, and R. Rahmadi, "Developing FB chatbot based on deep learning using RASA framework for university enquiries," arXiv, 2020.

[15] T. Bocklisch, J. Faulkner, N. Pawlowski, and A. Nichol, "Rasa: Open source language understanding and dialogue management," arXiv, pp. 19, 2017.

[16] L. Gunasekara and K. Vidanage, "UniOntBot: Semantic Natural Language Generation based API approach for Chatbot Communication," 2019 Natl. Inf. Technol. Conf. NITC 2019, pp. 8-10, 2019, doi: 10.1109/NITC48475.2019.9114440.

[17] M. Bagchi, "Conceptualising a library chatbot using open source conversational artificial intelligence," DESIDOC J. Libr. Inf. Technol., vol. 40, no. 6, pp. 329-333, 2020, doi: 10.14429/djlit.40.6.15611.

[18] B. Y. Folitse, J. Sam, L. P. Dzandu, and S. K. Osei, "Poultry Farmers' Information Needs and Sources in Selected Rural Communities in the Greater Accra Region, Ghana,” Int. Inf. Libr. Rev., vol. 50, no. 1, pp. 112, 2018, doi: 10.1080/10572317.2017.1351020.

[19] J. A. Khobragade, V. V. Banthiya, S. P. Landge, A. P. Dhok, M. M. Kadam, and J. D. Nandeshwar, "Information Need of Poultry Farmer from Vidarbha Region of Maharashtra," Int. J. Curr. Microbiol. Appl. Sci., vol. 8, no. 02, pp. 3373-3378, 2019, doi: 10.20546/ijcmas.2019. 802.392 .

[20] A. Sarkar, A. Goyal, D. Hicks, D. Sarkar, and S. Hazra, "Android Application Development: A Brief Overview of Android Platforms and Evolution of," 2019 Third Int. Conf. I-SMAC (IoT Soc. Mobile, Anal. Cloud), pp. 73-79, 2019.

[21] S. Saengwong and C. Koksantia, "Development of a mobile app for enhancing the performance of smallholder native chicken management and production," Asia-Pacific J. Sci. Technol., vol. 25, 2020.

[22] Rakesh Kumar Sharma, "An Analytical Study and Review of open source Chatbot framework, Rasa," Int. J. Eng. Res., vol. V9, no. 06, pp. 10111014, 2020, doi: 10.17577/ijertv9is060723.

[23] D. Braun, A. H. Mendez, F. Matthes, and M. Langen, "Evaluating natural language understanding services for conversational question answering systems," SIGDIAL 2017 - 18th Annu. Meet. Spec. Interes. Gr. Discourse Dialogue, Proc. Conf., no. August, pp. 174-185, 2017, doi: 10.18653/v1/w17-5522.

[24] K. Deepika, V. Tilekya, J. Mamatha, and T. Subetha, "Jollity Chatbot- A contextual AI Assistant," Proc. 3rd Int. Conf. Smart Syst. Inven. Technol. ICSSIT 2020, no. Icssit, pp. 1196-1200, 2020, doi: 10.1109/ICSSIT48917.2020.9214076.

[25] S. Hwang, B. Kim, and K. Lee, A Data-Driven Design Framework for Customer Service Chatbot, vol. 11583 LNCS. Springer International Publishing, 2019. 